\title{
Apelin aggravates the migration and invasion of non- small cell lung cancer cells via YAP1
}

\author{
Wenchao Ma ${ }^{1,2 \#}$, Di Zhu ${ }^{1 \#}$, Tong Yu ${ }^{1,2}$, Junwen Xue ${ }^{1}$, Xiang Sun ${ }^{1}$, Guofang Zhang ${ }^{1}$, \\ Yumeng Hou ${ }^{1}$, Jinrui $\mathrm{Li}^{1}$, Yingzhun Chen ${ }^{3}$, Hongli Shan ${ }^{1,2,4^{*}}$, Haihai Liang ${ }^{1,2,4^{*}}$
}

\begin{abstract}
Background: Apelin, an endogenous ligand of G-protein coupled receptor (GPCR), is a secreted peptide involved in the development of various tumors. However, the relationship between apelin and nonsmall cell lung cancer (NSCLC) is not quite clear. This study was designed to investigate the effect and mechanism of apelin on cell proliferation, migration and invasion of NSCLC cells. Methods: Twelve NSCLC specimens were collected for hematoxylin-eosin (HE) staining and immunohistochemistry analyses. Cell proliferation was examined by 3-(4,5-Dimethylthiazol-2-yl)-2,5-diphenyltetrazolium bromide (MTT) and cell migration and invasion were assessed using wound-healing and transwell assays. The subcellular location of yes associated protein 1 (YAP1) in A549 cells was determined by immunofluorescence. The mRNA and protein levels in NSCLC tissues and cell lines were measured by qRT-PCR and western blot, respectively. Results: Apelin was upregulated in tumor tissues compared with the adjacent tissues. Apelin promoted proliferation, migration, and invasion of A549 and $\mathrm{H} 460$ cells, which was reversed by competitive apelin receptor (APJ) antagonist ML221. Additionally, apelin upregulated YAP1 expression, whereas silence of YAP1 by small interfering RNA (siRNA) attenuated apelin-induced cell proliferation, migration and invasion and suppressed epithelial-mesenchymal transition progression. Conclusion: Apelin promotes NSCLC cells proliferation, migration, and invasion by modulating YAP1 and might be a potential therapeutic target for NSCLC treatment.
\end{abstract}

\section{Keywords}

non-small cell lung cancer ; apelin; yes associated protein 1 ; epithelial-mesenchymal transition

Received 27 January 2021, accepted 11 October 2021

\author{
State-Province Key Laboratories of Biomedicine-Pharmaceutics of China, Key \\ Laboratory of Cardiovascular Research, Ministry of Education,Department of \\ Pharmacology, College of Pharmacy, Harbin Medical University, Harbin 150081, \\ China \\ ${ }^{2}$ Northern Translational Medicine Research and Cooperation Center, Heilongjiang \\ Academy of Medical Sciences, Harbin Medical University, Harbin 150081, China \\ ${ }^{3}$ Department of Pathology, the 2nd Affiliated Hospital, Harbin Medical University, \\ Harbin 150081, China \\ ${ }^{4}$ Research Unit of Noninfectious Chronic Diseases in Frigid Zone (2019RU070), \\ Chinese Academy of Medical Sciences, Harbin 150081, China
}

${ }^{\text {\#} A u t h o r s ~ m a d e ~ e q u a l ~ c o n t r i b u t i o n ~ t o ~ t h i s ~ s t u d y ~}$

${ }^{*}$ Corresponding authors Prof. Haihai Liang, E-mail: lianghaihai@ems.hrbmu.edu.cn; Prof. Hongli Shan, E-mail: shanhongli@ems.hrbmu.edu.cn

\section{Introduction}

Lung cancer has long been considered the leading cause of cancer-related deaths worldwide ${ }^{[1]}$. Non-small cell lung cancer (NSCLC) accounts for over $80 \%$ of lung cancer cases, with more than $50 \%$ of adenocarcinoma and $30 \%$ of squamous carcinoma ${ }^{[2]}$. Targeted therapy is the best choice for patients with unreplaceable lung cancer with driver gene mutation ${ }^{[3]}$. In recent years, a variety of molecular targeted drugs have been put into clinical treatment, such as epidermal growth factor receptor (EGFR) inhibitor erlotinib for EGFR mutant cancer, anti-human epidermal growth factor receptor-2 (HER2) antibody trastuzumab for HER2 gene-amplified cancer ${ }^{[4]}$. However, these drugs cannot be effective for all types of cancer. It is of great importance to uncover novel mechanisms behind NSCLC metastasis and target molecules for developing new therapies.

Apelin, a secreted peptide, has been identified as the endogenous ligand of the G-protein-coupled cell-surface receptor apelin receptor $(A P J)^{[5]}$. Both apelin and its receptor APJ were considered to be key regulators of central and peripheral responses to various homeostatic perturbations. Numerous reports showed that apelin plays a role in regulating cardiovascular function, angiogenesis, fluid homeostasis and energy metabolism ${ }^{[6]}$. We have found that apelin enhanced $I_{K 1} l$ Kir2.1 current via the PI3K pathway to reverse ischemia- and hypoxia-induced depolarization of resting membrane potential (RMP) and prolongation of QT interval, indicating that apelin may 
play a role in preventing acute ischemic-mediated arrhythmias ${ }^{[7]}$. Previous studies also suggest apelin as an important factor in cancer $^{[5]}$. Moreover, the expression of apelin was found directly related to the capillaries size and the number of microvessels in NSCLC ${ }^{[8]}$. Accumulating data suggest that apelin/APJ might be a new pathway for molecular targeted therapy of cancers or as a potential diagnostic biomarker of cancer ${ }^{[9]}$.

Hippo signaling is an evolutionarily conserved network that coordinates cell proliferation and apoptosis to control organ development, tissue homeostasis and cancer ${ }^{[10]}$. When Hippo signaling is inactivated, yes-associated protein (YAP) and transcriptional co-activator with PDZ-binding motif (TAZ), two homologous transcriptional co-activator proteins that shuttle between the cytosol (phosphorylated inactive state) and cell nuclei (unphosphorylated active state), accumulate in the nucleus, and interact with the transcription co-factor TEA domain (TEAD). TEAD is one of the final nuclear effectors of the Hippo pathway, which activates gene transcription to promote growth and migration ${ }^{[11]}$. The activation of YAP can induce cancer stem cell-characteristics, proliferation, chemoresistance, and metastasis, making them attractive therapeutic targets for cancer $^{[12]}$. A recent study from our group confirmed that YAP1 promotes Slug transcription via TEAD contributing to NSCLC invasion and migration ${ }^{[13]}$. However, the upstream regulatory mechanism of YAP1 in NSCLC remains unclear.

Here, we reported apelin can promote the development of nonsmall cell lung cancer, and depletion of YAP1 decreased the capacity of tumor metastasis that enhanced by apelin. Apelin can be regarded as a potential target to overcome tumor metastasis in NSCLC.

\section{Materials and Methods}

\subsection{Patients and specimens}

NSCLC tissue specimens were obtained from 12 patients undergoing surgical procedures in the Second Affiliated Hospital of Harbin Medical University between December 2018 and March 2020. Prior to the use of the samples, the patients were all provided with informed written consents and the study protocol involving the human samples was approved by the Harbin Medical University Ethic Review Committees (NO. IRB300720).

\subsection{Immunohistochemistry}

Immunohistochemistry (IHC) staining was used to determine the expression levels of apelin in tumor and non-tumor tissues. The tissue sections of $5-\mu \mathrm{M}$ thick on slides were deparaffinized in xylene and rehydrated in graded alcohol solutions. The antigen recovery process was performed by heating the sections in TrisEDTA buffer for $30 \mathrm{~min}$. Then the slides were incubated with apelin antibody (Elabscience, Wuhan, China; $1: 100$ ) at $4^{\circ} \mathrm{C}$ overnight. The sections were then stained with the respective secondary antibodies, followed by counterstaining with hematoxylin, dehydration and mounting. Images were captured by a Zeiss microscope (Carl Zeiss, Oberkochen, Germany).

\subsection{Cell culture}

The NSCLC cell lines A549, H1299 and H460 were purchased from the Cell Bank of the Chinese Academy of Sciences (Shanghai, China). Cells were cultured in Roswell Park Memorial Institute (RPMI) 1640 (Biological Industries, Kibbutz BeitHaemek, Israel) supplemented with $10 \%$ fetal bovine serum (FBS, Biological Industries, Kibbutz Beit-Haemek, Israel) and $1 \%$ penicillin/streptomycin at $37^{\circ} \mathrm{C}$ and $5 \% \mathrm{CO}_{2}$.

\subsection{Transfection}

To specifically knockdown YAP1 expression, small interfering RNA (siRNA) specifically targeting YAP1 were purchased from Sigma (Sigma-Aldrich, St. Louis, MO, USA). Cells were transfected with siRNA using Lipofectamine 2000 (Invitrogen, Carlsbad, CA, USA) according to the manufacturer's instructions ${ }^{[14]}$, and a scramble oligonucleotide was used as a negative control. After 6 to $8 \mathrm{~h}$, the cells were incubated with 1 $\mu \mathrm{mol} / \mathrm{L}$ apelin for $48 \mathrm{~h}$.

\subsection{Wound-healing assay}

Wound-healing assay was performed to assess cell migration. Cells were seeded at a density of $1 \times 10^{6}$ cells/well in six-well plates. An artificial wound was created on the confluent cell monolayer using a sterile $10-\mu \mathrm{L}$ pipette tip. The cells were washed with phosphate buffer saline (PBS) to remove cell debris. The wounds were photographed with a light microscope at 0,24 , and $48 \mathrm{~h}$ after treatment.

\subsection{Transwell assay}

Transwell migration and invasion assay was performed according to the standard method ${ }^{[15]}$. The number of migrated cells in five randomly selected fields was counted under a light microscope (Olympus, Tokyo, Japan). All experiments were repeated at least three times.

\subsection{Immunofluorescence staining}

$1 \times 10^{6}$ cells were seeded on coverslips in 24 -well plates. After 
transfection and apelin treatment, the cells were fixed in cold methanol for $20 \mathrm{~min}$ and washed with PBS. Then, the cells were permeabilized with $0.1 \%$ Triton $\mathrm{X}-100$ for $1 \mathrm{~h}$ and washed thoroughly with PBS, followed by blocking with goat serum at room temperature for $40 \mathrm{~min}$. Next, the cells were incubated with primary antibodies overnight and subsequently incubated with Alexa Fluor 488-tagged or Alexa Fluor 594-tagged secondary antibodies (Life Technologies). After washing with PBS, the nuclei were stained with DAPI (Beyotime Biotechnology, Shanghai, China).

\subsection{RNA extraction and quantitative real-time PCR}

Total RNA was isolated from the tissues with TRIzol reagent (Invitrogen, Carlsbad, CA, USA) according to the manufacturer's instructions ${ }^{[16]}$. The amount of RNA was quantified with a NanoDrop 8000 Spectrophotometer (Thermo Scientific, Wilmington, DE, USA). cDNA was generated using the High Capacity cDNA Reverse Transcription Kit (Applied Biosystems, Foster City, CA, USA). Real-time PCR was performed on an ABI7500 FAST real-time PCR System (Applied Biosystems) for 40 cycles. After the reaction cycles, the threshold cycle $(\mathrm{Ct})$ values were determined, and the relative mRNA levels were calculated based on the $\mathrm{Ct}$ values that were normalized to the glyceraldehyde3-phosphate dehydrogenase (GAPDH) level in each sample. Primer sets for apelin (5'-GATGCCGCTTCGATG-3'/5'ATTCCTTGACCCTCTGGGCT-3') were provided by Invitrogen (Carlsbad, CA, USA).

\subsection{Western blot}

Cells were lysed with radio immunoprecipitation assay (RIPA) Iysis buffer (Beyotime Biotechnology, Shanghai, China) with a protease inhibitors cocktail. Approximately $100 \mu \mathrm{g}$ of crude protein was run on $10 \%$ SDS-PAGE gels for electrophoretic separation, which were then transferred onto nitrocellulose membranes (Merck Millipore, R7BA46025). The blots were probed with primary antibodies for. FN-1 (Proteintech Group, Wuhan, China; 1:1000), E-cadherin (Proteintech Group, Wuhan, China; 1:500), Vimentin (CST, MA, USA; 1:1000), and GAPDH as an internal control (Proteintech Group, Wuhan, China; 1:5 $000)$ in PBS at $4^{\circ} \mathrm{C}$ overnight. The membranes were washed with PBS-Tween 20 and then incubated with secondary antibody (Alexa Fluor) at room temperature for $1 \mathrm{~h}$. Finally, the western blot bands were collected with an imaging system (Odyssey, LICOR, USA) and quantified by measuring the intensity in each group with Odyssey v1.2 Software (Odyssey, LICOR, USA).

\subsection{MTT assay}

Cell suspensions were seeded in 96-well culture plates for $12 \mathrm{~h}$ post transfection. MTT of $20 \mu \mathrm{L}(5 \mathrm{mg} / \mathrm{mL})$ was added to each well $(180 \mu \mathrm{L}$ medium) and cells were maintained in the dark at $37{ }^{\circ} \mathrm{C}$ with $5 \% \mathrm{CO}_{2}$ for $4 \mathrm{~h}$. After aspiration of the medium, 150 $\mu \mathrm{L}$ of dimethyl sulfoxide (DMSO) was added to each well, and the resultant precipitate was dissolved by pipetting up and down for $15 \mathrm{~min}$. The absorbance of this solution was measured at a wavelength of $490 \mathrm{~nm}$.

\subsection{Statistical analysis}

The data were statistically analyzed using GraphPad Prism 8.0 software (GraphPad Software, San Diego, CA, USA) and are expressed as the mean \pm SEM. One way analysis of variance (ANOVA) followed by Bonferroni or Dunnett's post-hoc test was used for multiple group comparisons. The difference was considered as statistically significant $(P<0.05)$.

\section{Results}

\subsection{Apelin expression is upregulated in NSCLC}

Hematoxylin and eosin (HE) staining assay was performed to distinguish the tumorous tissue from the adjacent area (Fig. 1A). To investigate the possible clinical significance of apelin in NSCLC, we examined the expression of apelin using IHC assay and found that apelin expression was obviously higher in NSCLC tissues than in the paired adjacent tissues (Fig. 1B). Consistently, qRT-PCR and western blot assays demonstrated that both the mRNA and protein levels of apelin were dramatically upregulated in NSCLC tissues compared with those in the paired adjacent tissues (Fig. 1C and Fig. 1D). We further evaluated apelin expression on various NSCLC cell lines A549, H460, and H1299, and found that there was no significant difference in the expression of apelin in the above three cells (Fig. 1E).

\subsection{Apelin promotes NSCLC cells proliferation, migration and invasion}

To identify the potential role of apelin on NSCLC progression, we tested three concentrations $(0.5,1$, and $2 \mu \mathrm{mol} / \mathrm{L})$ of apelin for its effects on NSCLC proliferation and migration using MTT and wound-healing assays in A549 cells (Fig. 2). Based the results, apelin at a concentration of $1 \mu \mathrm{mol} / \mathrm{L}$ was used for the subsequent experiments.

Next, we used A549 and H460 cells to examine the effect of apelin on several features of cancer cells. MTT assay showed that $1 \mu \mathrm{mol} / \mathrm{L}$ apelin treatment enhanced cell viability in both A549 and H460 cells (Fig. 3A and Fig. 3B). Then, we tested the autonomous migration ability of A549 and $\mathrm{H} 460$ cells by using wound-healing assay. As shown in Fig. 3C and Fig. 3D, apelin 
A

B
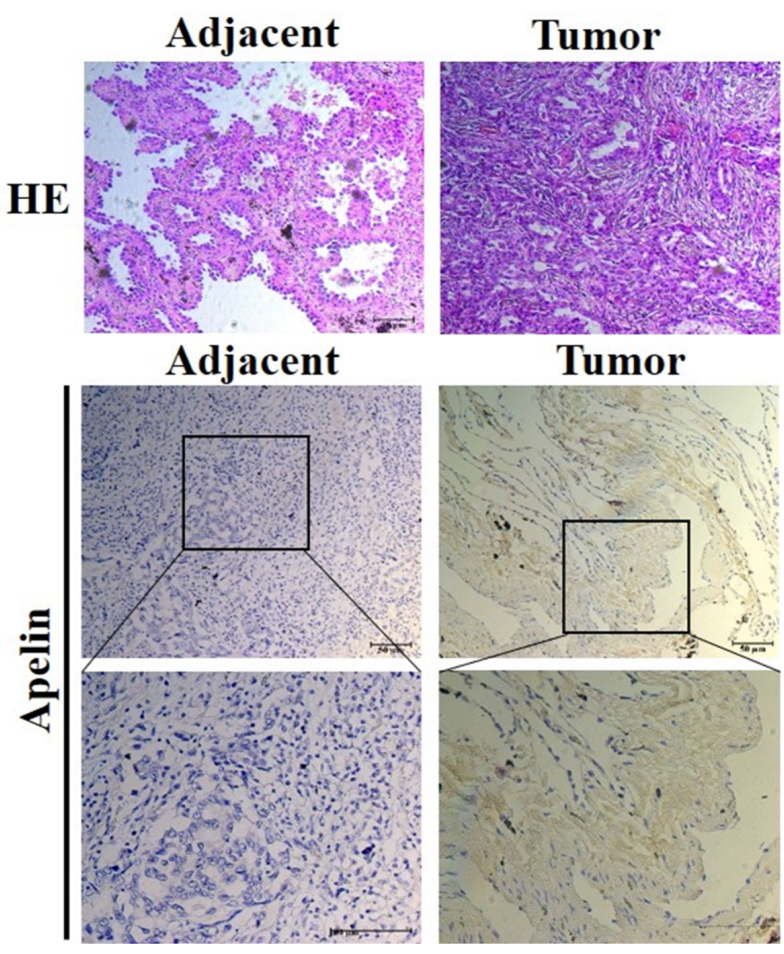

Tumor

E

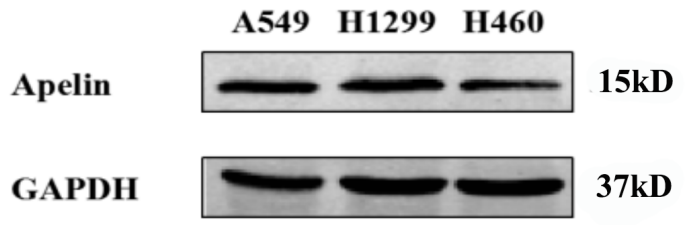

C

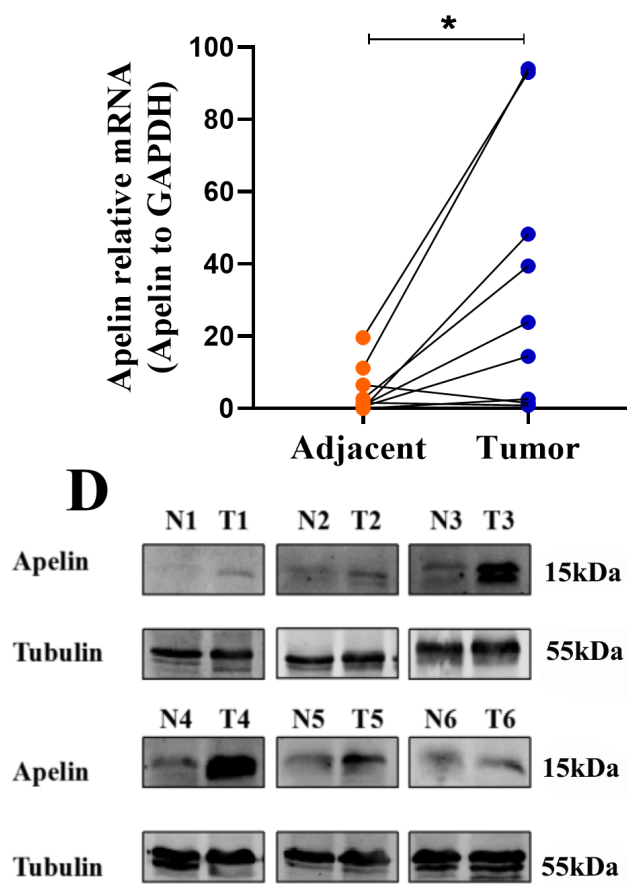

Adjacent Tumor

Fig. 1 Exaggerated expression of apelin in NSCLC specimens

(A) HE staining of human NSCLC tissues and matched adjacent tissues. (B) Representative IHC imaging of apelin in NSCLC tissues. Scale bar, $50 \mu$ mol/L. (C) qRT-PCR analysis of apelin mRNA levels in human NSCLC tissues and paired adjacent tissues. $N=8,{ }^{*} P<0.05$. (D) Representative western-blot outcomes of apelin in 12 pairs of normal (N) and tumor (T) samples of NSCLC; Scatter diagram on the right panel represents relative apelin expression. $N=12,{ }^{*} P<0.05$. (E) Western-blot assay of apelin protein levels in three NSCLC cell lines was performed. 
A

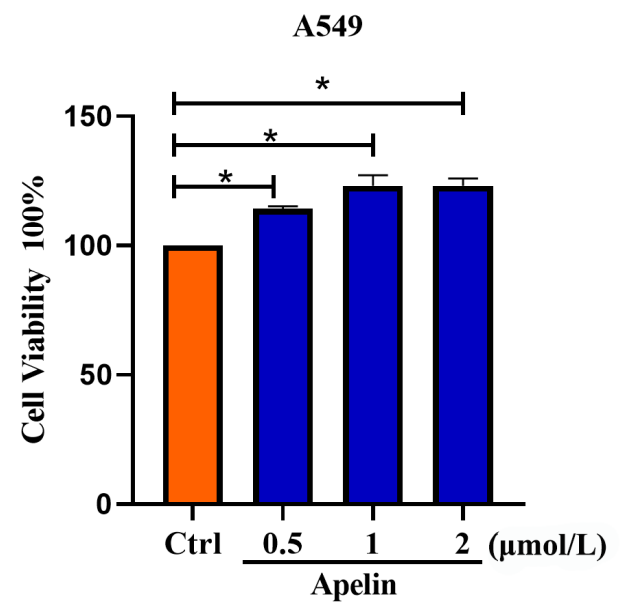

C
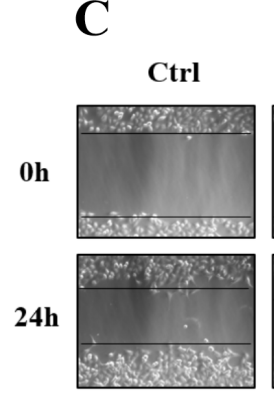

$48 \mathrm{~h}$

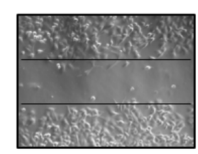

$\operatorname{Apelin}(\mu \mathrm{mol} / \mathrm{L})$
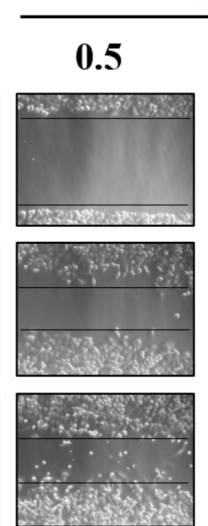

B

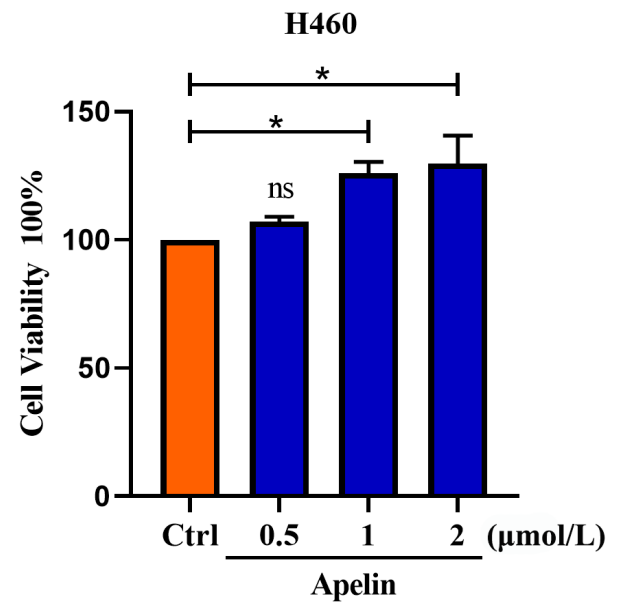

A549

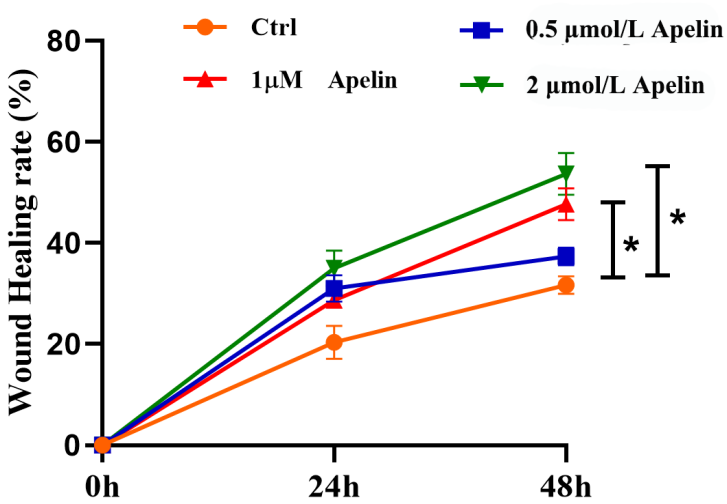

Fig. 2 Effects of different concentrations of apelin on NSCLC

Effect of apelin on cell viability was evaluated by MTT assay in A549 (A) and H460 (B) cells. $N=5$, * $P<0.05$. (C) The wound-healing assay were used to identify the promigration effect of apelin in A549 cells. $N=3,{ }^{*} P<0.05$. 
A

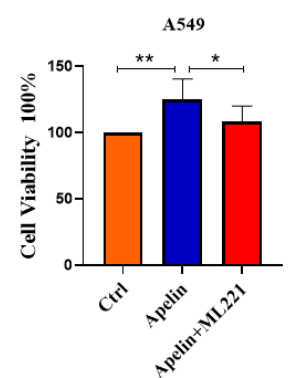

C

0h

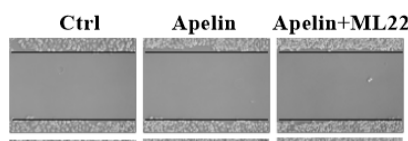

$24 h$

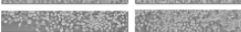

$48 \mathrm{~h}$

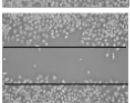

D

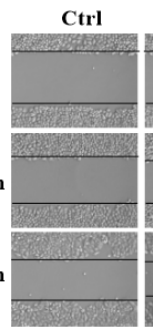

$\mathbf{E}$
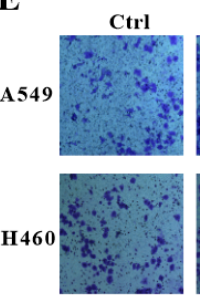

F

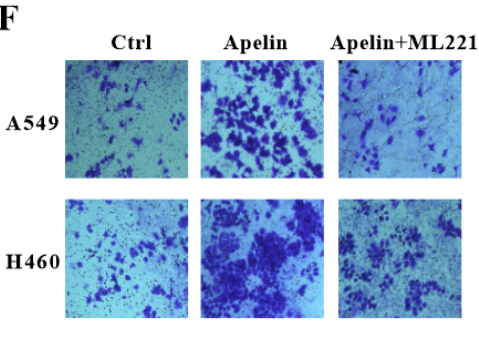

B
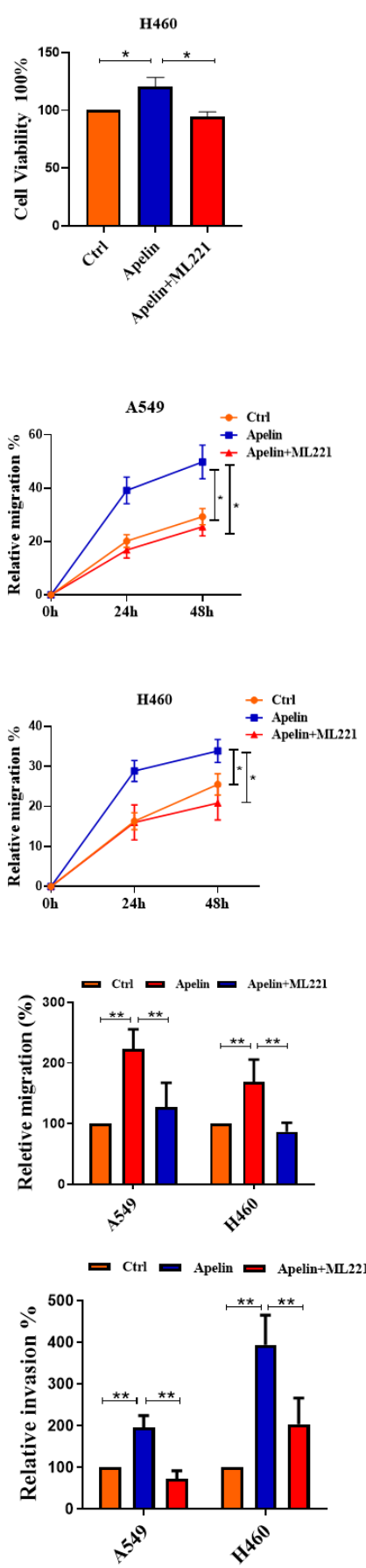

Fig. 3 Apelin promotes A549 and H460 cells proliferation, migration and invasion via APJ

MTT analysis of cell viability in A549 (A) and H460 (B) cells with apelin and APJ antagonist ML221. Wound-healing assays for A549 cells (C) and H460 cells (D) with apelin and ML221 at $0,24,48 \mathrm{~h}$ after scratching. $N=6,{ }^{*} P<0.05,{ }^{*} P<0.01$. Transwell assay detected the migration (E) and invasion (F) of A549 and H460 cells with apelin and ML221. Cell counts were for the corresponding assays of at least four random microscope fields. $N=3,{ }^{* *} P<0.01$. 
significantly accelerated the rate of wound closure in both A549 and $\mathrm{H} 460$ cells. Consistently, transwell assay further confirmed that apelin promoted cell migration and invasion (Fig. 3E and Fig. 3F).

It is known that apelin can activate the apelin receptor APJ. We used ML221, a functional antagonist of APJ, which binds to APJ and blocks the activation of APJ. We found that ML221 alleviated apelin-induced increases in cell viability, migration, and invasion in both $\mathrm{A} 549$ and $\mathrm{H} 460$ cells (Fig. 3).

\subsection{Apelin activates YAP1 and promotes its expression in nucleus}

Having clarified the role of apelin in A549 and H460, we set out to explore the molecular mechanism. We have previously demonstrated that YAP1 regulates the transcription of Slug in a TEAD-dependent manner and promotes NSCLC tumorigenesis ${ }^{[13]}$. We therefore proposed that apelin might regulate YAP1-induced epithelial-mesenchymal transition (EMT) in NSCLC. To examine the hypothesis, we conducted immunofluorescence analysis and found that apelin upregulated YAP1 expression, especially in nucleus (Fig. 4A and Fig. 4B). Moreover, western-blot results also showed that apelin evidently increased the protein level of YAP1 (Fig. 4C and Fig. 4D).

\subsection{Apelin aggravates cell migration and invasion via YAP1}

To investigate whether YAP1 mediates the effect of apelin on NSCLC cells migration and invasion, we used siRNA to silence the expression of endogenous YAP1. MTT analysis demonstrated that YAP1 siRNA abolished the proliferationpromoting effect of apelin (Fig. 5A and Fig. 5B). Wound healing assay confirmed that apelin markedly promoted cell migration, whereas silence of YAP1 abrogated this effect. (Fig. 5C and Fig. 5D). Moreover, the results from transwell assay revealed that apelin increased the migration and invasion of A549 and $\mathrm{H} 460$ cells, while knockdown of YAP1 reduced the migratory and invasive capacity of NSCLC cells (Fig. 6A and Fig. 6B). These results support that YAP1 mediates the oncogenic property or more specifically the NSCLC-promoting action of apelin.

\subsection{Apelin induced EMT progression by YAP1}

To clarify whether apelin promoted EMT by regulating YAP1, we examined the effect of apelin on EMT in both A549 and H460 cells with or without YAP1 siRNA. Western blot analysis showed that the expression of epithelial marker E-cadherin was decreased, whereas that of mesenchymal markers vimentin and FN-1 was increased by apelin, which was reversed by YAP1 siRNA (Fig. 6A and Fig. 6B). Consistent with this results, immunofluorescence assays showed that apelin inhibited the expression of Zo-1 and increased the expression of vimentin, whereas these effect was alleviated after silencing YAP1 (Fig. 6C and Fig. 6D).

\section{Discussion}

In the present study, we showed that apelin is a key regulator of EMT and promotes the migration and invasion of NSCLC cells. We demonstrated that inhibited YAP1 expression are associated with alleviated EMT and NSCLC progression. Apelin overexpression promotes the nuclear translocation of YAP1. Moreover, apelin increased the migration and invasion of A549 and $\mathrm{H} 460$ cells by activating YAP1. Taken together, these results indicate that apelin inhibition could be a novel strategy for the treatment of NSCLC.

APJ is a member of the seven-transmembrane $G$ protein-coupled receptor (GPCR) family, and apelin is an endogenous ligand of APJ and its C-terminal is the specific binding region of the APJ receptor ${ }^{[17-18]}$. Apelin triggers intracellular signaling pathway by binding and activating its receptor APJ ${ }^{[6]}$. The apelin/APJ pathway is found in many organs including heart, blood vessels, lung, liver, brain and kidney ${ }^{[19]}$ where it plays a role in regulating cell proliferation, apoptosis, glucose metabolism, infecting cardiac function and angiogenesis. Apelin also regulates the secretion of certain hormones, aging process, and fluid homeostasis and produces neuroprotective effects ${ }^{[20-22]}$. Some reports revealed that apelin stimulated the formation of migratory protrusions and affected actin cytoskeleton rearrangement and the proteolytic abilities of colon cancer cells ${ }^{[23]}$. The PAK1-cofilin pathway mediates migration of human adenocarcinoma cells induced by apelin/APJ ${ }^{[24]}$. According to the latest evidence, the expression of apelin/APJ is increased in numerous cancers, such as colon adenomas and adenocarcinomas, ovarian cancer, cholangiocarcinoma, human hepatocellular carcinoma ${ }^{[25]}$. However, the exact mechanism of apelin in regulating NSCLC is unclear. Therefore, further study is warranted to examine whether apelin could act as a predictor of NSCLC. In this study, we examined the clinical samples from NSCLC patients and found that the expression of apelin in NSCLC tumor tissues was significantly higher than in the adjacent tissues. In vitro experiments proved that apelin promoted the proliferation, invasion, and migration of NSCLC cell lines A549 and H460. Moreover, we found that ML221 alleviated apelin-induced cell proliferation, migration, and invasion in both A549 and H460 cells. All these results suggest that apelin might be a potential target for treatment of lung cancer.

Hippo signaling has been proven to contribute to the progression of various diseases including cancer. Extensive research implicates that the Hippo pathway is dysregulated in numerous 
A

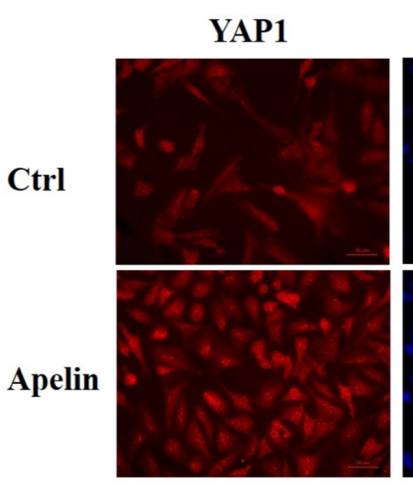

B
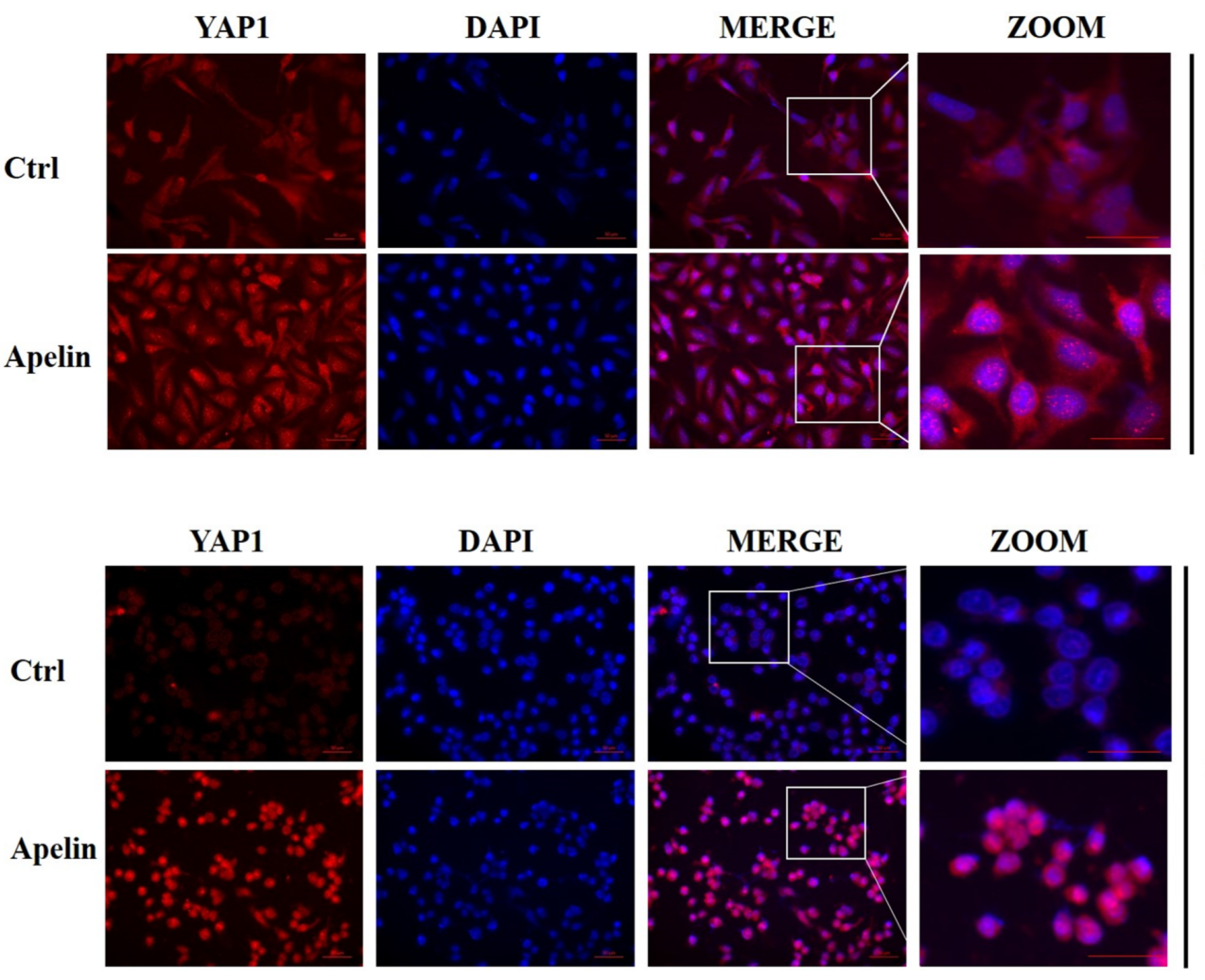

A549
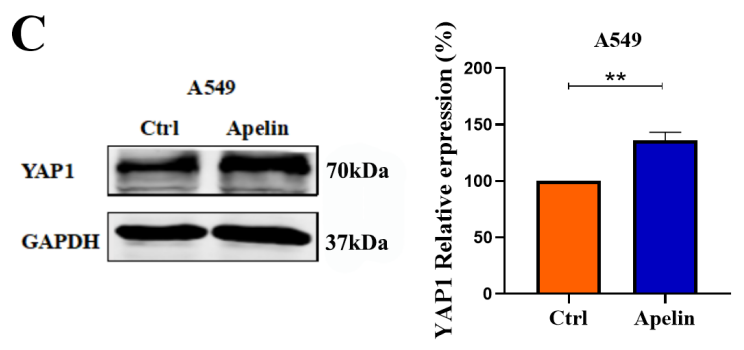

D
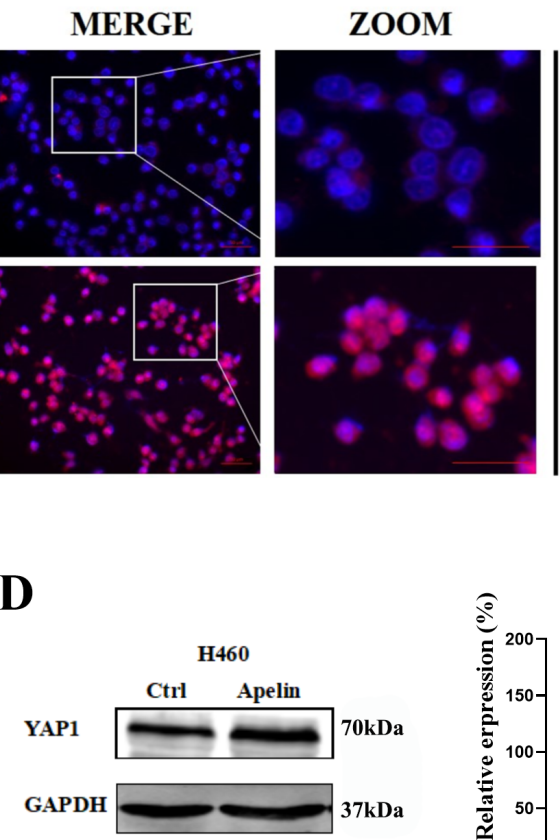

H460

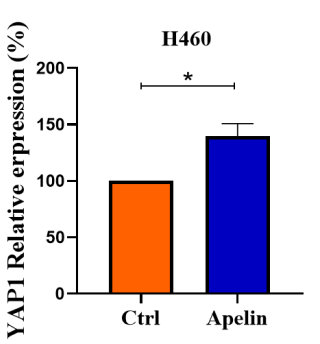

Fig. 4 Apelin activates YAP1 signaling

(A and B) Immunofluorescence assays showed that the apelin upregulated YAP1 expression in nuclear in A549 and H460 cells. (C and D) Western-blot analysis showing the expression of YAP1 in the presence of apelin treatment in A549 and H460 cells. $N=6,{ }^{*} P<0.05,{ }^{* *} P<0.01$. 
A

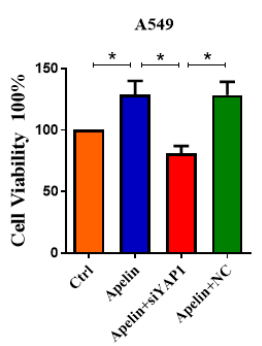

oh

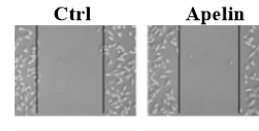

$24 \mathrm{~h}$
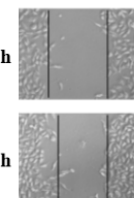

D
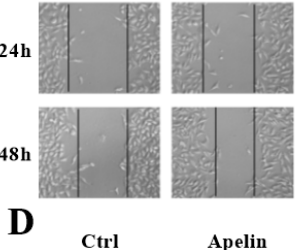

oh
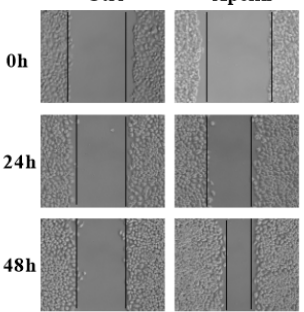

E
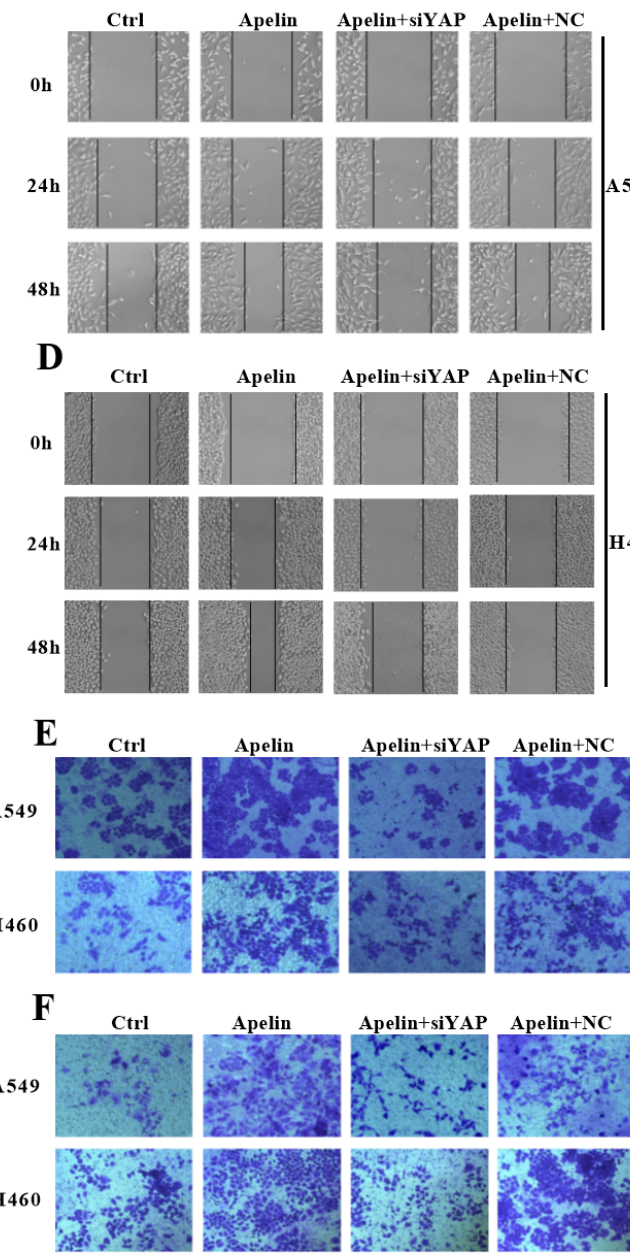

B
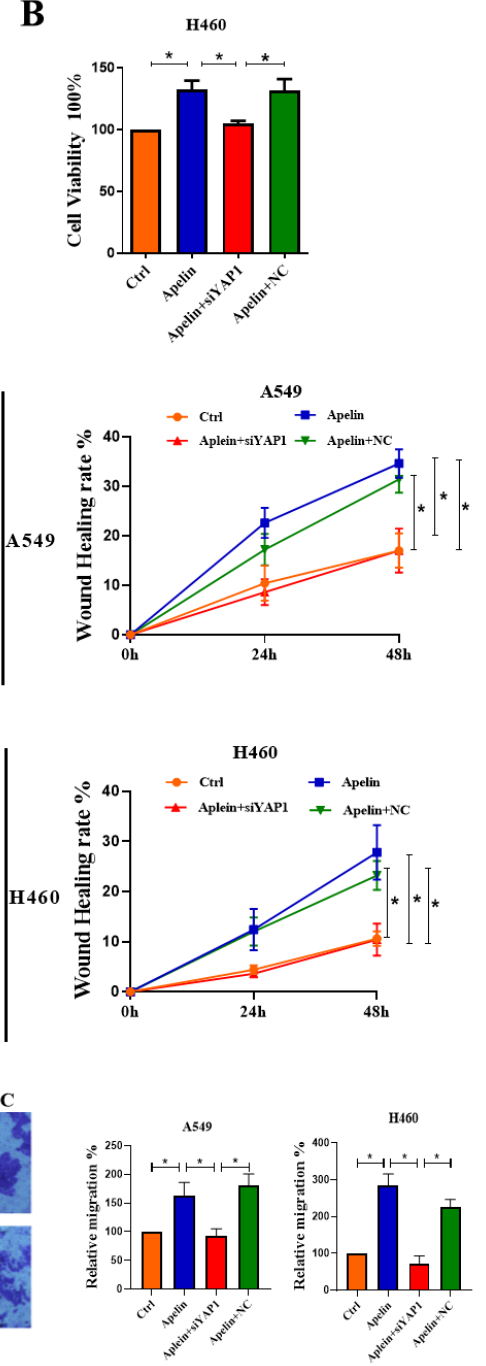
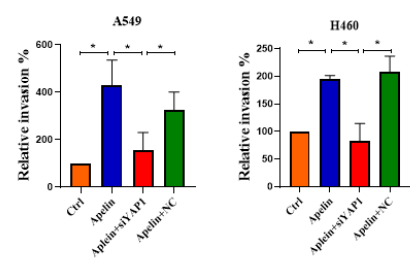

\section{Fig. 5 Apelin regulates cell migration and invasion through YAP1}

Cell viability in A549 (A) and $\mathrm{H} 460$ (B) cells with YAP1 silencing. $N=6,{ }^{*} P<0.05$. Wound-healing assays of A549 (C) and H460 (D) cells with YAP1 silencing at 0,24 , $48 \mathrm{~h}$ after scratching. $N=6,{ }^{*} P<0.05$. The migration and invasion abilities of A549(E) and H460 (F) cells after transfection with YAP1 siRNA. Cell counts were for the corresponding assays of at least four random microscope fields. $N=3,{ }^{*} P<0.05$. 
A

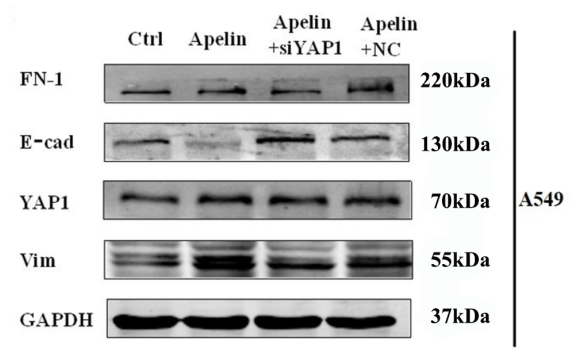

B

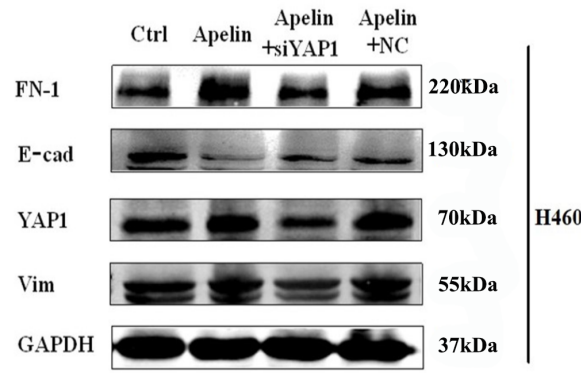

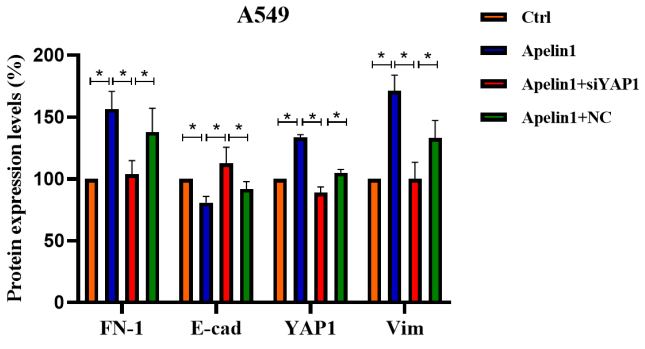

H460

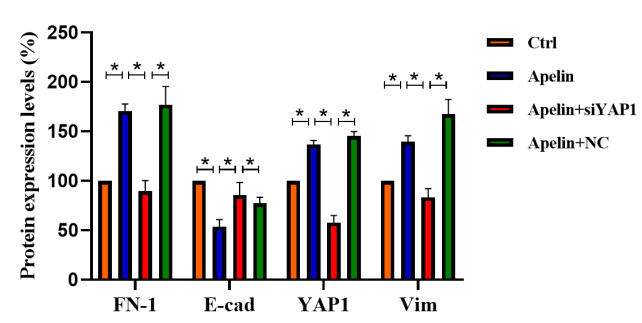

C
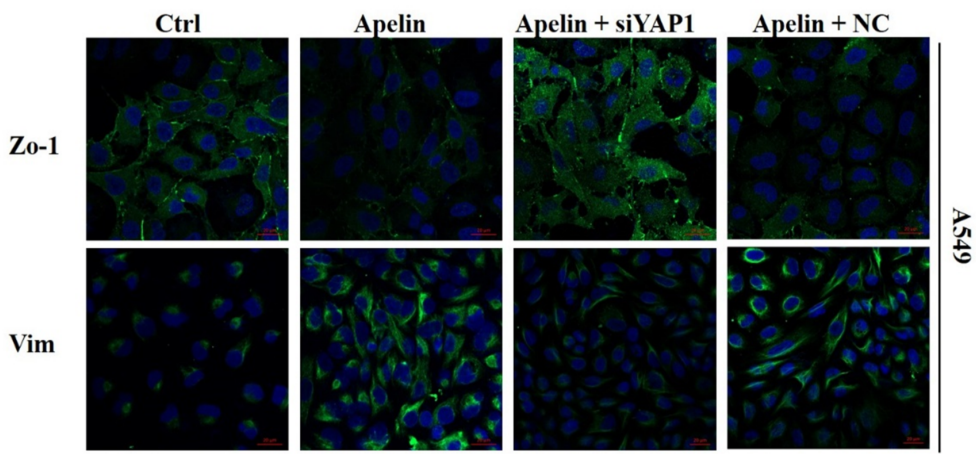

D

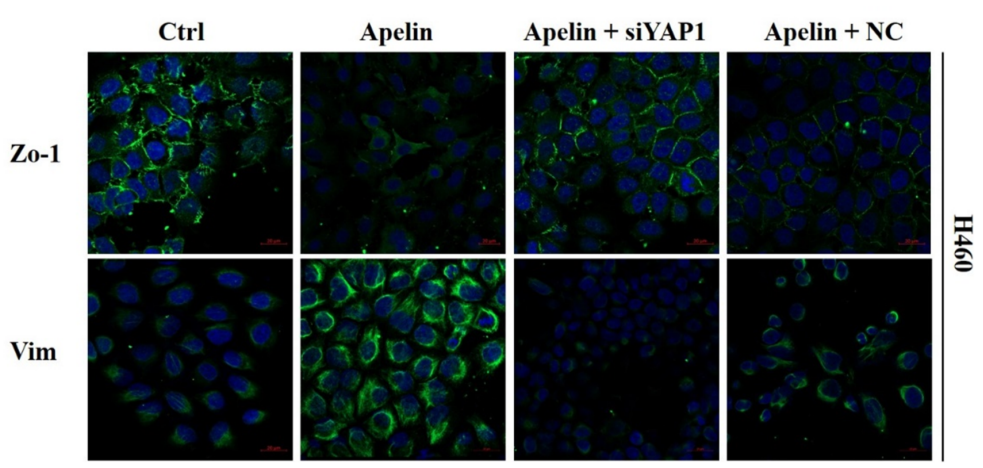

Fig. 6 Apelin regulates EMT program by modulating YAP1

(A and B) Western-blot analysis showed the expression of EMT-relevant proteins (FN-1, E-cadherin, Vimentin) after knockdown of YAP1 in the presence of apelin treatment. $N=6$, ${ }^{*} P<0.05$. (C and D) Effect of YAP1 on apelin induced EMT was determined by immunofluorescence in H460 and A549 cells. 
human cancers across the body ${ }^{[26]}$. In clear cell renal cell carcinoma (ccRCC), knockdown of YAP inhibited the cell cycle process and increased cell apoptosis ${ }^{[27]}$. The overexpression of YAP/TAZ is closely related to the development, progression and poor prognosis of NSCLC ${ }^{[28]}$. The YAP/TAZ proteins are highly enriched in the nucleus of actively proliferating cells in a dephosphorylated form, which can enhance fundamental cellular functions, including cell proliferation and tumorigenesis ${ }^{[29]}$. In this study, we found that apelin increased YAP1 protein expression, especially in nucleus.

EMT is an important process in promoting the invasion and metastasis of cancer. Epithelial cells lose epithelial characteristics and obtain a mesenchymal, highly invasive phenotype during the EMT progression ${ }^{[30]}$. Loss of cell polarity is a hallmark of cancer and an initial step in EMT, a transdifferentiation program whereby epithelial cell acquired a more spread, migratory and fibroblast-like shape. As oncoproteins, YAP/TAZ promote cell proliferation, transformation, and cancer cell stemness ${ }^{[31]}$. Overexpression of YAP in human non-transformed mammary epithelial cells induced EMT, and upregulation of TAZ expression in mammary cells leads to the acquisition of a spindle-shaped morphology and increased cell migration and invasion ${ }^{[32]}$. The canonical mechanism for the action of YAP is the regulation of a phosphorylated kinase cascade of the Hippo pathway downstream GPCR, Kibra, TA01/2/3 kinase, AMPK and MAPK signaling ${ }^{[33]}$. Abnormal GPCR signaling is an important mechanism of cancer development ${ }^{[34]}$. Studies have found that GPCRs are powerful inducers of the YAP carcinogenic pathways ${ }^{[35]}$. And recent studies suggested that the Hippo pathway was a downstream branch of GPCR signaling. GPCRs mediated signals can positively or negatively modulate YAP/TAZ activity, which depended on the nature of signals, receptors, and adaptor proteins ${ }^{[10,36]}$. We have demonstrated for the first time that YAP1 promoted NSCLC tumorigenesis by regulating the transcription of Slug ${ }^{[13]}$. Therefore, we assume apelin can regulate EMT progress through YAP1. As we expected, epithelial marker E-cadherin was decreased and mesenchymal markers FN-1 and vimentin was increased after the treatment of apelin, while knockdown YAP1 decreased the EMT program of A549 and H460 cells induced by apelin.

In the recent years, the Hippo pathway has attracted much attention due to its role in organ size control and tumorigenesis. GPCRs are the largest cell membrane receptor family, which can regulate a series of downstream signaling mediators, including Hippo pathway effectors YAP/TAZ. For cancers with a dependency on high YAP/TAZ activity, drugs targeting GPCRs and $G$ proteins may reduce YAP/TAZ activation and slow the progression of cancer ${ }^{[37]}$. The GPCR-Hippo signaling axis may become a promising target for anti-cancer therapy, but it still needed further research. Our studies show that apelin related to the carcinogenesis and cancer metastasis, providing a new target for the establishment of novel anti-cancer therapy.

People in cold regions are susceptible to polycyclic aromatic hydrocarbons (PAH) that can be produced by burning coal for keeping warm. Inhaled PAH mainly distributed in the alveoli, and the metabolites of PAH may alter DNA replication and transcription which probably induce neoplasms, thus leading to elevated risk of getting lung cancer.

Taken together, this study demonstrated that apelin promoted the proliferation, migration and invasion progression of NSCLC cells through inducing EMT process via regulating YAP1. In addition, interfering with apelin expression might be a novel strategy for the prevention and treatment of NSCLC.

\section{Conflicts of interests}

The authors declare no competing interests.

\section{Author contributions}

$\mathrm{H}-\mathrm{HL}$ and $\mathrm{H}-\mathrm{LS}$ designed the research. D.Z. and $\mathrm{W}-\mathrm{CM}$ performed the experiments and drafted the manuscript. J-WX, G-FZ, J-RL and Y-MH performed cellular experiments. L-FL, $X S$ and $Y-Z C$ provided the samples and pathological analysis. D.Z., T.Y and H-LS analyzed the data. All authors have read and approved the final manuscript.

\section{Acknowledgements}

This study was supported by the National Natural Science Foundation of China (81872863) and the Major Scientific Fund Project of Heilongjiang Province (ZD2019H001).

\section{Ethics approval and consent to participate}

Prior to the use of the samples, the patients were all provided with informed written consents and the study protocol involving the human samples was approved by the Harbin Medical University Ethic Review Committees (NO. IRB300720). 


\section{References}

[1] Bray F, Ferlay J, Soerjomataram I, et al. Global cancer statistics 2018: GLOBOCAN estimates of incidence and mortality worldwide for 36 cancers in 185 countries. CA Cancer J Clin, 2020; 70(4): 313.

[2] Zhang $M$, Yang W, Wang P, et al. CCL7 recruits $\mathrm{CDC} 1$ to promote antitumor immunity and facilitate checkpoint immunotherapy to non-small cell lung cancer. Nat Commun, 2020; 11(1): 6119.

[3] Kwak E L, Bang Y J, Camidge D R, et al. Anaplastic lymphoma kinase inhibition in non-small-cell lung cancer. N Engl J Med, 2010; 363(18): 1693-1703.

[4] Hamanaka N, Nakanishi Y, Mizuno T, et al. YES1 is a targetable oncogene in cancers harboring YES1 gene amplification. Cancer Res, 2019; 79(22): 5734-5745.

[5] Sorli S C, Le Gonidec S, Knibiehler B, et al. Apelin is a potent activator of tumour neoangiogenesis. Oncogene, 2007; 26(55): 76927699 .

[6] O'Carroll A M, Lolait S J, Harris L E, et al. The apelin receptor APJ: journey from an orphan to a multifaceted regulator of homeostasis. $J$ Endocrinol, 2013; 219(1): R13-35.

[7] Li Z, He Q, Wu C, et al. Apelin shorten QT interval by inhibiting Kir2.1/ IK1 via a PI3K way in acute myocardial infarction. Biochem Bioph Res Co, 2019; 517(2): 272-277.

[8] Berta J, Kenessey I, Dobos J, et al. Apelin expression in human nonsmall cell lung cancer: role in angiogenesis and prognosis. J Thorac Oncol, 2010; 5(8):1120-1129.

[9] Yang Y, Lv S Y, Ye W, et al. Apelin/APJ system and cancer. Clin Chim Acta, 2016; 457: 112-116.

[10] Yu F X, Zhao B, Guan K L. Hippo pathway in organ size control, tissue homeostasis, and cancer. Cell, 2015; 163(4): 811-828.

[11] Zhang Z, Du J, Wang S, et al. OTUB2 promotes cancer metastasis via hippo-independent activation of YAP and TAZ. Mol Cell, 2019; 73(1): 7-21 e7.

[12] Zanconato F, Piccolo S. Eradicating tumor drug resistance at its YAP-biomechanical roots. EMBO J, 2016; 35(5): 459-461.

[13]Yu M, Chen Y, Li X, et al. YAP1 contributes to NSCLC invasion and migration by promoting Slug transcription via the transcription co-factor TEAD. Cell Death Dis, 2018; 9(5): 464.

[14] Liang $\mathrm{H}, \mathrm{Yu}$ T, Han $\mathrm{Y}$, et al. LncRNA PTAR promotes EMT and invasion-metastasis in serous ovarian cancer by competitively binding miR-101-3p to regulate ZEB1 expression. Mol Cancer, 2018; 17(1): 119.

[15] Wu M, Duan Q, Liu X, et al. MiR-155-5p promotes oral cancer progression by targeting chromatin remodeling gene ARID2. Biomed Pharmacother, 2020; 122: 109696.

[16] Li X, Yu T, Shan $\mathrm{H}$, et al. IncRNA PFAL promotes lung fibrosis through CTGF by competitively binding miR-18a. FASEB J, 2018; 32(10): 5285-5297.

[17] Tatemoto K, Hosoya M, Habata Y, et al. Isolation and characterization of a novel endogenous peptide ligand for the human APJ receptor. Biochem Bioph Res Co, 1998; 251(2): 471-476.

[18] Cano Martinez L J, Coral Vazquez R M, Mendez J P, et al. Serum concentrations of apelin-17 isoform vary in accordance to blood pressure categories in individuals with obesity class 3. Clin Exp Hypertens, 2019; 41(2): 168-173.
[19] Yan J, Wang A, Cao J, et al. Apelin/APJ system: an emerging therapeutic target for respiratory diseases. Cell Mol Life Sci, 2020; 77(15): 2919-2930.

[20] Wu L, Chen L, Li L. Apelin/APJ system: a novel promising therapy target for pathological angiogenesis. Clin Chim Acta, 2017; 466: 78-84.

[21] Xie $H$, Yuan $L$ Q, Luo X H, et al. Apelin suppresses apoptosis of human OBs. Apoptosis, 2007; 12(1): 247-254.

[22] Yu X H, Tang Z B, Liu L J, et al. Apelin and its receptor APJ in cardiovascular diseases. Clin Chim Acta, 2014; 428: 1-8.

[23] Podgorska M, Pietraszek-Gremplewicz K, Nowak D. Apelin effects migration and invasion abilities of colon cancer cells. Cells, 2018; 7(8): 113.

[24] Lv D, Li L, Lu Q, et al. PAK1-cofilin phosphorylation mediates human lung adenocarcinoma cells migration induced by apelin-13. Clin Exp Pharmacol Physiol, 2016; 43(5): 569-579.

[25] Masoumi J, Jafarzadeh A, Khorramdelazad H, et al. Role of Apelin/ APJ axis in cancer development and progression. Adv Med Sci, 2020; 65(1): 202-213.

[26] Plouffe S W, Hong A W, Guan K L. Disease implications of the Hippo/ YAP pathway. Trends Mol Med, 2015; 21(4): 212-222.

[27] Cao J J, Zhao X M, Wang D L, et al. YAP is overexpressed in clear cell renal cell carcinoma and its knockdown reduces cell proliferation and induces cell cycle arrest and apoptosis. Oncol Rep, 2014; 32(4): 15941600.

[28] Malik S A, Khan M S, Dar M, et al. Molecular alterations and expression dynamics of LATS1 and LATS2 genes in non-small-cell lung carcinoma. Pathol Oncol Res, 2018; 24(2): 207-214.

[29] Pan D. The hippo signaling pathway in development and cancer. Dev Cell, 2010; 19(4): 491-505.

[30] Mao X Y, Li Q Q, Gao Y F, et al. Gap junction as an intercellular glue: Emerging roles in cancer EMT and metastasis. Cancer Lett, 2016; 381(1): 133-137.

[31] Dong J, Feldmann G, Huang J, et al. Elucidation of a universal sizecontrol mechanism in drosophila and mammals. Cell, 2007; 130(6): 11201133.

[32] Cordenonsi M, Zanconato F, Azzolin L, et al. The Hippo transducer TAZ confers cancer stem cell-related traits on breast cancer cells. Cell, 2011; 147(4): 759-772.

[33] Gao J, He L, Zhou L, et al. Mechanical force regulation of YAP by F-actin and GPCR revealed by super-resolution imaging. Nanoscale, 2020; 12(4): 2703-2714

[34] Dorsam R T, Gutkind J S. G-protein-coupled receptors and cancer. Nat Rev Cancer, 2007; 7(2): 79-94.

[35] Yu F X, Zhang Y, Park H W, et al. Protein kinase A activates the Hippo pathway to modulate cell proliferation and differentiation. Gene Dev, 2013; 27(11): 1223-1232.

[36] Yu F X, Mo J S, Guan K L. Upstream regulators of the Hippo pathway. Cell Cycle, 2012; 11(22): 4097-4098.

[37] Luo J, Yu F X. GPCR-Hippo Signaling in Cancer. Cells, 2019; 8(5): 426. 